\title{
The Effect of Glucose Added to the Dialysis Fluid on Blood Pressure, Vasoactive Hormones and Energy Transfer during Hemodialysis in Chronic Renal Failure
}

—An Analysis of Changes in Blood Pressure and Plasma Concentration of Renin, Angiotensin II, Endothelin, Insulin, Glucagon and Growth Hormone

\author{
Erling B. Pedersen*, Birte Ardal, Jesper N. Bech, Thomas G. Lauridsen, \\ Niels A. Larsen, Lisbeth Mikkelsen, Maren Sangill, Ingrid M. Thomsen \\ Department of Medical Research and Department of Medicine, Holstebro Hospital and \\ University of Aarhus, Aarhus, Denmark \\ E-mail: *ebp@dadlnet.dk \\ Received November 3, 2011; revised November 22, 2011; accepted December 8, 2011
}

\begin{abstract}
Background: Previous studies showed that blood pressure was reduced in patients with chronic renal failure during hemodialysis with glucose added to the dialysis fluid. We wanted to test the hypotheses that blood pressure is reduced in non-diabetic and diabetic dialysis patients, when glucose is added to the dialysis fluid, and that blood pressure changes are caused by changes in plasma concentrations of vasoactive hormones or to vasodilation secondary to an increase in body temperature. Methods: The effect of dialysis with glucose added to the dialysis fluid was measured in three randomized, placebo-controlled, un-blinded and cross-over studies with periods of one week duration. In non-diabetic nephropathy (Study $1, n=19$ ) and diabetic nephropathy (Study 2, $n=15$ ), we measured blood pressure (BP) and pulse rate (PR), plasma concentrations of glucose (p-Glucose), renin (PRC), angiotensin II (p-AngII), endothelin (p-Endot), insulin (p-Ins), glucagon (p-Glu), and human growth hormone (p-hGH). In non-diabetic nephropathy (Study 3, n = 24), we measured the effect of dialysis with glucose added to the dialysis fluid on energy transport from form the body using body temperature control. Results: Study 1 and 2 showed that BP, PRC, p-AngII, and p-Ins were unchanged, whereas P-Endot increased and P-hGH decreased, in dialysis patients with or without glucose added to the dialysis fluid. In diabetics, a marginal increase in p-Glu was measured during dialysis with glucose, but not without glucose. Study 3 showed that SBP increased significantly using dialysis with temperature control of dialysis fluid compared with no temperature control (145 versus $138 \mathrm{~mm} \mathrm{Hg}$ ). In parallel with the increase in SBP, the energy flux from the patients was significantly higher with temperature control than without. Conclusion: In non-diabetics and diabetics, blood pressure was unchanged during dialysis with glucose added to the dialysis fluid in a short-term study. Vasoactive hormones in plasma were changed in the same way independently of glucose in the dialysis fluid. Systolic blood pressure increased using dialysis with temperature control of dialysis fluid, presumably due to vasoconstriction to prevent or antagonize a fall in body temperature.
\end{abstract}

Keywords: Angiotensin, Blood Pressure, Chronic Renal Failure, Diabetes, Dialysis, Dialysis Fluid, Endothelin, Glucagon, Growth Hormone, Hemodialysis, Insulin, Renin

\section{Introduction}

Patients with chronic renal failure often have such adverse reactions as fatigue, headache, disorientation and malaise during and in the hours after a dialysis session. This can be due to manifest or undiagnosed hypoglycae- mia [1,2], since $15 \mathrm{~g}$ - $30 \mathrm{~g}$ of glucose is removed during a dialysis session [3-6]. Hypoglycaemia can be prevented or the incidence can be decreased by addition of glucose to the dialysis fluid [4-6], and the occurrence of headache and fatigue is reduced $[5,7,8]$. However, in some studies dialysis patients do not become hypoglycaemic 
during treatment with glucose-free dialyses fluid [5,9].

Previously, we have performed a randomized, placebo controlled, un-blinded, cross-over study of haemodialysis patients, who were allocated to treatment with and without glucose in the dialysis fluid during two 10 weeks periods [10]. We found that blood pressure was reduced when glucose was added to the dialysis fluid, but the underlying mechanism for the blood pressure reduction was not clarified.

Plasma insulin increases, when the glucose concentration in the dialysis fluid exceeds pre-dialysis concentration of blood glucose [5,9]. An increase in plasma insulin within the physiological range results in vasodilatation in both animals and man [11,12]. A fall in blood pressure will be antagonized by a reflex mediated increased in the sympathetic adrenergic activity. In our previous study the pulse rate was unchanged, although blood glucose increased. Thus, the explanation seems to be more complex, partly because patients with chronic renal failure have some degree of insulin resistance, and consequently also of insulin induced vasodilatation [13], and partly due to the fact that the response to pressure factors/hormones generally is decreased in chronic renal failure. An alternative explanation of the blood pressure reduction could be an increase in metabolism due to the glucose load and subsequently an increase in body temperature, vasodilatation and fall in blood pressure.

New knowledge regarding the pathophysiological mechanism during dialysis treatment with glucose added to the dialysis fluid may have consequences for future clinical practice in treatment of patients with chronic renal failure.

We wanted to test the hypotheses that blood pressure is reduced in non-diabetic and diabetic dialysis patients, when glucose is added to the dialysis fluid, that blood pressure changes can be attributed to changes in plasma concentrations of vasoactive hormones (renin, angiotensin II, endothelin, insulin, glucagon and human growth hormone), and that blood pressure changes are related to an increased glucose metabolism with an increase in body temperature and subsequently vasodilatation when glucose is added to dialysis fluid.

The purpose was to measure the effect of dialysis with glucose added to the dialysis fluid in three randomized, placebo-controlled, un-blinded and cross-over studies. In Study 1 of dialysis patients with non-diabetic nephropathy, we measured 1) Blood pressure (BP) and pulse rate (PR), 2) Plasma concentration of glucose (p-Glucose), 3) Plasma concentrations of renin (PRC), angiotensin II (pAngII), and Endothelin (p-Endot), 4) Plasma concentrations of insulin (p-Ins), glucagon (p-Glu), human growth hormone (p-hGH), and 5) Energy transport from the body. In Study 2 of dialysis patients with diabetic nephropathy, we measured the same effect variables as in Study 1. In Study 3 of dialysis patients with non-diabetic nephropathy, we measured the effect of dialysis with glucose added to the dialysis fluid on energy transport from form the body using body temperature control.

\section{Material and Methods}

\subsection{Design}

All three studies were randomized, placebo-controlled, unblinded, and cross-over. During the active treatment period, patients received treatment with hemodialysis with glucose added to the dialysis fluid. During the placebo period, patients received treatment without glucose added to the dialysis fluid. Each study was of 4 weeks duration. Week 1was a "run-in" period, and week 3 was a "wash-out" period. During weeks 2 and 4, patients received dialysis treatment with dialysis with and without glucose added to the dialysis fluid after randomization.

\subsection{Ethical Aspects}

The studies were approved by the local Ethics Committee and Danish Data Protection Agency. The study was done according to the rules given in Good Clinical Practice. The three studies were registered in ClinicalTrials. gov Protocol Registration System under the numbers: NCT 00438295, NCT 00438503, and NCT 00439023.

\subsection{Patients}

\section{Group 1: Non-diabetic nephropathy}

Inclusion criteria: Age $>18$ years, both men and women, and chronic renal failure treated with haemodialysis for more than 3 months.

Exclusion criteria: Heart failure, lung insufficiency, liver disease, diabetes mellitus, other endocrine diseases than diabetes mellitus not sufficiently treated, malignant disease, nephrotic syndrome, treatment with haemodiafiltration, other diseases or conditions which implied that a patient could not participate, and unwillingness to participate.

Withdrawal criteria: Development of one of the exclusion criteria, changes in dialysis treatment during the study period, and kidney transplantation.

Group 2: Diabetic nephropathy

The inclusion, exclusion and withdrawal criteria were as in group 1 except that diabetes mellitus was added as an inclusion criterion and removed as exclusion criterion.

\subsection{Recruitment}

All patients were recruited among dialysis patients in the 
Department of Medicine, Holstebro Hospital.

\subsection{Number of Patients}

The minimum relevant difference in SBP was estimated to $8 \mathrm{~mm} \mathrm{Hg}$. The standard deviation was estimated to be $9 \mathrm{~mm} \mathrm{Hg}$. With a level of significance of $5 \%$ and a power of $80 \%, 16$ participants should be needed.

\subsection{Effect Variables}

The primary effect variables was a change in systolic BP. The other effect variables were PR, b-Glucose, PRC, pAngII, p-Endot, p-Ins, p-Glu, p-hGH and energy transfer during dialysis treatment.

\subsection{Treatment}

Treatment with and without glucose added to the dialysis fluid was given as active and placebo treatment respectively.

\subsection{Experimental Procedure}

Patients were informed according to the rules of the local Ethics Committee and gave written consent to participate. Randomization to treatment with glucose added to or not added to the dialysis fluid in either week 2 or week 4 . In week 1 ("run-in") dialysis treatment was given according to the routine procedure in the department three times a week. In week 2 (dialysis with or without glucose added to the dialysis fluid according to the randomization), dialysis treatment was given as in week 1 . Blood pressure, pulse rate, p-glucose and body temperature were determined at dialysis start and every hour during each dialysis session. Energy transport to or from the patient was measured at each dialysis session. At the third dialysis in this period, blood samples were drawn for measurements of hormones at dialysis start, and after two and four hours, i.e. the end of the dialysis session. In week 3 ("wash out"), dialysis treatment was given as in week 1 . In week 4 (dialysis with or without glucose added to the dialysis fluid according to the randomization), dialysis treatment, monitoring and blood sampling were as in week 2 .

\section{Methods}

Hemodialysis, blood pressure and pulse rate, and energy flux

Hemodialysis was performed using Hemodialysis Machines 4008 H/S (Fresenius Medical Care). Automatic measurement of blood pressure and pulse rate were done, using the principle of oscillometry in a Blood Pressure Monitor (BPM). Automatic body temperature control was obtained and thermal energy flux calculated using a Blood Temperature Monitor (BTM). BPM and BTM were accessories to the dialysis machine.

Glucose

Blood glucose was determined using a HemoCue B-glucose Analyzer, based on a glucose dehydrogenase method for whole blood.

\section{Hormones}

Blood samples were centrifuged for 15 minutes at 3000 $\mathrm{rpm}$ at $4^{\circ} \mathrm{C}$. Plasma was separated from blood cells and kept frozen at $-80^{\circ} \mathrm{C}$ until assayed.

PRC was determined using an immunoradiometric assay from CIS Biointernational, Gif-Sur-Yvette Cedex, France. The coefficients of variation were $0.9 \%-3.6 \%$ (intra-assay) and $3.7 \%-5.0 \%$ (inter-assay) in the range 4-263 pg/ml. The detection limit was $1 \mathrm{pg} / \mathrm{ml}$. Normal range (supine) was $1.2-20.2 \mathrm{pg} / \mathrm{ml}$ in subjects older than 40 years.

P-Ang II was extracted from plasma with $\mathrm{C}_{18}$ Sep-Pak (Water associates, Milford, MA, USA), and subsequently determined by radioimmunoassay [14]. The antibody against Ang II was obtained from Department of Clinical Physiology, Glostrup Hospital, Denmark. Minimal detection level was $2 \mathrm{pmol} / \mathrm{L}$. The coefficients of variation were $12 \%$ (inter-assay) and $8 \%$ (intra-assay). Normal range was $4.6-18.4 \mathrm{pg} / \mathrm{ml}$.

P-Endot was determined using a human endothelin-1 immunoassay from R \& D systems, Minneapolis, USA. The coefficients of variation were $4.2 \%$ - $4.6 \%$ (intra-assay) and $5.1 \%$ - $6.6 \%$ (inter-assay) in the range $14-70 \mathrm{pg} / \mathrm{ml}$. The detection limit was less than $1.0 \mathrm{pg} / \mathrm{ml}$. Normal range was $0.3-0.9 \mathrm{pg} / \mathrm{ml}$

P-Ins was determined using a human insulin specific RIA kit from Linco Research, St. Charles, Missouri, USA. The coefficients of variation were $3.1 \%$ - $4.4 \%$ (intra-assay) and $2.9 \%-6.0 \%$ (inter-assay) in the range $8-54 \mu \mathrm{U} / \mathrm{ml}$. The sensitivity was $2 \mu \mathrm{U} / \mathrm{ml}$ using a $100 \mu \mathrm{l}$ sample size. Normal fasting range was $5-15 \mu \mathrm{U} / \mathrm{ml}$.

P-Glu was determined using a glucagon RIA kits from Linco Research, St. Charles, Missouri, USA. The coefficients of variation were $4.0 \%-6.8 \%$ (intra-assay) and $7.3 \%$ - 13.5\% (inter-assay) in the range $60-220 \mathrm{pg} / \mathrm{ml}$. The sensitivity was $20 \mathrm{pg} / \mathrm{ml}$ using a $100 \mu \mathrm{l}$ sample size. Normal fasting range was $50-150 \mathrm{pg} / \mathrm{ml}$.

P-hGH was determined as hGH-RIACT using an immunoradiometric assay from CIS Biointernational, GifSur-Yvette Cedex, France. The coefficients of variation were $1.3 \%-2.1 \%$ (intra-assay) and 3.8\% - 5.0\% (interassay) in the range $4-63 \mu \mathrm{IU} / \mathrm{ml}$. The detection limit was $0.03 \mu \mathrm{IU} / \mathrm{ml}$. Normal range was $0-28.5 \mu \mathrm{IU} / \mathrm{ml}$ with $93 \%$ bekow $15 \mu \mathrm{IU} / \mathrm{ml}$.

\section{Statistics}

SPSS was used. A General Linear Model for repeated 
measurement was used for the statistical analyses for comparisons with-in and between groups. Wilcoxon's signed rank test was used for paired comparison between two groups. Mann-Whitney's test was used for unpaired comparison between two groups. Data are presented as medians with 25 and 75 percentiles. Significance level is 0.05 .

\section{Results}

\subsection{Demographics}

Study 1 comprised 19 non-diabetic patients with chronic renal failure treated with haemodialysis, mean age of 67 years, range 21 - 88, 12 men and 7 women. Study 2 comprised 15 diabetic patients with chronic renal failure treated with haemodialysis, mean age 63 years, range 36 - 80, 10 men and 5 women, type 1 diabetes in 8 and type 2 diabetes in 7 . Study 3 comprised 24 non-diabetic patients with chronic renal failure treated with haemodialysis, mean age 76 years, range 26 - 86, 15 men and 9 women. Patients were on a standard treatment regimen with a combination of B-vitamins (pyridoxine, riboflavin, thiamin, dexpanthenol, nicotinamide), vitamin C, and folic acid. All patients were treated with dialysis three times weekly to obtain a Kt/V greater than 1.2.

In the three groups studied the following values were measured at inclusion in Groups 1, 2 and 3 respectively: B-Hemoglobin (mmol/l): $7.4 \pm 0.7 ; 7.4 \pm 0.6$; $7.5 \pm 0.6$. P-Creatinine $(\mu \mathrm{mol} / \mathrm{l}): 608 \pm 152 ; 727 \pm 164 ; 611 \pm 154$. P-Carbamide (mmol/l): $19 \pm 5 ; 22 \pm 6 ; 17 \pm 4$. P-Ca ${ }^{++}$ (mmol/l): $1.24 \pm 0.08 ; 1.18 \pm 0.07 ; 1.20 \pm 0.08$. P-Phosphate (mmol/l): $1.54 \pm 0.45 ; 1.51 \pm 0.30 ; 1.52 \pm 0.48$. PParathyroid hormone (pmol/l): $20 \pm 14 ; 20 \pm 10 ; 22 \pm 18$. Hemoglobin A1c: $0.054 \pm 0.003 ; 0.069 \pm 0.006 ; 0.054 \pm$ 0.004. P-Albumin (g/l): $39 \pm 3 ; 40 \pm 4$; $37 \pm 8$.

The following medication was used. Erythropoietin in 46 patients, iron saccharose in 29 , calcium carbonate in 30 , sevelamer in 20 , alfacalcidol in 32 , cina- calcet in 4 , $\beta$-adrenoceptor blockers in 23, calcium channel blockers in 16, ACE-inhibitors in 20, angiotensin II re- ceptor blockers in 16, diuretics in 24 and minoxidil in 10 .

\subsection{Blood Pressure and Pulse Rate}

Both systolic and diastolic blood pressure was the same in non-diabetics and diabetics during dialysis sessions both with and without dialysis with glucose (Table 1). In diabetics, the pulse rate was significantly higher during dialysis without glucose, whereas no difference was measured in the non-diabetics. The effect of dialysis was the same on SBP, DBP and pulse rate in both type 1 and 2 diabetics (Table 2).

\subsection{Blood Glucose Concentration}

Blood glucose was significantly higher during dialysis with glucose both in non-diabetics and diabetics (Table 1).

\subsection{Plasma Concentrations of Renin, Angiotensin II and Endothelin}

In both non-diabetics and diabetics, PRC and p-AngII were unchanged during dialysis treatment with and without glucose and at the same level (Table 3). P-Endot increased markedly during dialysis in both non-diabetics and diabetics during dialysis with and without glucose from approximately 1.1 - 1.3 to $1.3-1.6 \mathrm{pg} / \mathrm{ml}$.

Both in Type 1 and Type 2 diabetics, PRC and p-AngII were unchanged during dialysis treatment with and without glucose and in the same level, but p-Endot increased significantly in both types (Table 4).

Table 1. Systolic and diastolic blood pressure (SBP, DBP), pulse rate (PR), blood glucose (b-glucose), and energy transfer during hemodialysis treatment using dialysis fluid with glucose (active treatment) and without glucose (placebo) in non-diabetic nephropathy and diabetic nephropathy in a randomized, placebo controlled, crossover study during four consecutive weeks (Week 1: run-in; Week 2: active treatment or placebo; Week 3: wash-out; Week 4: active treatment or placebo).

\begin{tabular}{lcccccc}
\hline & \multicolumn{2}{c}{ Non-diabetic nephropathy $(\mathrm{N}=19)$} & \multicolumn{3}{c}{ Diabetic nephropathy $(\mathrm{N}=15)$} \\
\hline & With glucose & Without glucose & $\mathrm{p}$ & With glucose & Without glucose & $\mathrm{p}$ \\
\hline SBP (mm Hg) & $137(125-148)$ & $138(120-149)$ & 0.841 & $136(128-150)$ & $134(124-149)$ & 0.609 \\
DBP (mm Hg) & $69(64-78)$ & $67(65-79)$ & 0.936 & $66(63-75)$ & $69(62-76)$ & 0.161 \\
PR (beats/min) & $72(67-80)$ & $73(67-78)$ & 0.872 & $66(62-83)$ & $76(72-93)$ & 0.001 \\
B-glucose (mmol/l) & $7.1(6.9-7.9)$ & $5.9(5.6-6.3)$ & 0.000 & $7.5(6.1-8.9)$ & $6.6(5.9-7.6)$ & 0.018 \\
Energy transfer(KJ) & -123 & -125 & 0.494 & -91 & -77 & $((-102)-(-8))$ \\
\hline
\end{tabular}

Data presented as medians with quartiles in brackets. For each dialysis session mean was calculated based on 5 - 8 measurements. Mean values of these variables from the three weekly dialysis sessions are indicated. Wicoxon's signed rank test was used for statistical analysis. 
Table 2. Systolic and diastolic blood pressure (SBP, DBP), pulse rate (PR), blood glucose (b-glucose) and energy transfer during hemodialysis treatment using dialysis fluid with glucose (active treatment) and without glucose (placebo) in diabetic nephropathy type 1 and diabetic nephropathy type 2 in a randomized, placebo controlled, crossover study during four consecutive weeks (Week 1: run-in; Week 2: active treatment or placebo; Week 3: wash-out; Week 4: active treatment or placebo).

\begin{tabular}{lcccccc}
\hline & \multicolumn{2}{c}{ Diabetic nephropathy type 1 $(\mathrm{N}=8)$} & \multicolumn{3}{c}{ Diabetic nephropathy type 2 (N=7) } \\
\hline & With glucose & Without glucose & $p$ & With glucose & Without glucose & p \\
\hline SBP (mm Hg) & $134(124-159)$ & $134(120-161)$ & 0.889 & $141(128-148)$ & $134(124-148)$ & 0.499 \\
DBP (mmHg) & $69(57-84)$ & $70(57-87)$ & 0.180 & $66(64-72)$ & $69(63-74)$ & 0.345 \\
PR (beats/min) & $74(63-91)$ & $82(73-102)$ & 0.012 & $65(70-88)$ & $76(70-88)$ & 0.018 \\
B-glucose (mmol/l) & $7.6(6.3-8.9)$ & $7.1(6.3-7.9)$ & 0.109 & $7.5(5.9-9.4)$ & $6.4(5.4-6.6)$ & 0.068 \\
Energy transfer(KJ) & $-81((-128)-(-12))$ & $-111((-12)-(-87))$ & 0.327 & $-58(-115-73)$ & $-15(-102-73)$ & 0.735 \\
\hline
\end{tabular}

For each dialysis session means were calculated for SBP, DBP, PR, and B-glucose based on 5 - 8 measurements. Mean values of these variables from the three weekly dialysis sessions were subsequently calculated. These data are presented as medians with quartiles in brackets. Wicoxon's signed rank test was used for statistical analysis.

Table 3. Plasma concentrations of renin (PRC), angiotensin II (p-AngII), and endothelin (p-Endot) during hemodialysis treatment using dialysis fluid with glucose (active treatment) and without glucose (placebo) in non-diabetic nephropathy and diabetic nephropathy in a randomized, placebo controlled, crossover studies during four consecutive weeks (Week 1: run-in; Week 2: active treatment or placebo; Week 3: wash-out; Week 4: active treatment or placebo).

\begin{tabular}{|c|c|c|c|c|c|c|c|c|}
\hline & \multicolumn{4}{|c|}{ Non-diabetic nephropathy (N = 19) } & \multicolumn{4}{|c|}{ Diabetic nephropathy $(\mathrm{N}=15)$} \\
\hline & Start & After 2 hours & End & P (GLM-with-in) & Start & After 2 hours & End & P (GLM-with-in) \\
\hline \multicolumn{9}{|c|}{ PRC (pg/ml) } \\
\hline With glucose & $10(5-41)$ & $11(6-37)$ & $11(4-37)$ & \multirow{3}{*}{0.269} & $13(7-50)$ & $20(9-69)$ & $16(11-62)$ & \multirow{3}{*}{0.146} \\
\hline Without glucose & $9(5-49)$ & $11(4-36)$ & $9(3-35)$ & & $15(9-55)$ & $25(11-49)$ & $23(5-56)$ & \\
\hline P (GLM-between) & & 0.897 & & & & 0.589 & & \\
\hline \multicolumn{9}{|c|}{ P-AngII (pg/ml) } \\
\hline With glucose & $5(2-7)$ & $6(4-11)$ & $7(3-12)$ & \multirow{3}{*}{0.967} & $2(1-17)$ & $4(2-18)$ & $5(2-17)$ & \multirow{3}{*}{0.397} \\
\hline Without glucose & $4(3-8)$ & $5(3-9)$ & $5(3-10)$ & & $3(1-21)$ & $4(2-21)$ & $6(2-23)$ & \\
\hline P (GLM-between) & & 0.811 & & & & 0.773 & & \\
\hline \multicolumn{9}{|c|}{ Endot $(\mathrm{pg} / \mathrm{ml})$} \\
\hline With glucose & $1.1(0.8-1.5)$ & $1.3 *(1.0-1.7)$ & $1.4 *(1.0-1.9)$ & \multirow[t]{3}{*}{0.000} & $1.3(0.8-1.5)$ & $1.4^{*}(1.0-1.6)$ & $1.5^{*}(1.0-1.8)$ & \multirow[t]{3}{*}{0.000} \\
\hline Without glucose & $1.2(0.9-1.4)$ & $1.5^{*}(1.0-2.0)$ & $1.3^{*}(1.0-1.9)$ & & $1.2(0.9-1.5)$ & $1.4^{*}(1.0-1.8)$ & $1.5 *(0.9-1.8)$ & \\
\hline P (GLM-between) & & 0.797 & & & & 0.991 & & \\
\hline
\end{tabular}

Data presented as medians with quartiles in brackets. Hormones were measured in the last dialysis session in Week 2 and Week 4 . Blood samples were drawn at dialysis start, after two hours and at the end of the session. A General Linear Model (GLM) for Repeated Measures was used for comparison within and between groups. Wilcoxon's signed rank test was used in each group to analyze significant deviations from baseline $(*=\mathrm{p}<0.05)$.

Table 4. Plasma concentrations of renin (PRC), angiotensin II (p-AngII), and endothelin (p-Endot) during hemodialysis treatment using dialysis fluid with glucose (active treatment) and without glucose (placebo) in Type 1 and Type 2 diabetic nephropathy in a randomized, placebo controlled, crossover studies during four consecutive weeks (Week 1: run-in; Week 2: active treatment or placebo; Week 3: wash-out; Week 4: active treatment or placebo).

\begin{tabular}{|c|c|c|c|c|c|c|c|c|}
\hline & \multicolumn{4}{|c|}{ Type 1 Diabetic nephropathy $(\mathrm{N}=8)$} & \multicolumn{4}{|c|}{ Type 2 Diabetic nephropathy $(\mathrm{N}=7$ ) } \\
\hline & Start & After 2 hours & End & P (GLM-with-in) & Start & After 2 hours & End & P (GLM-with-in) \\
\hline \multicolumn{9}{|c|}{ PRC (pg/ml) } \\
\hline With glucose & $25(8-26)$ & $28(11-88)$ & $23(12-71)$ & \multirow{2}{*}{0.381} & $10(5-50)$ & $11(5-69)$ & $12(5-54)$ & \multirow{3}{*}{0.083} \\
\hline Without glucose & $27(10-28)$ & $32(12-105)$ & $28(5-90)$ & & $12(7-34)$ & $25(6-49)$ & $23(5-42)$ & \\
\hline P (GLM-between) & & 0.729 & & & & 0.701 & & \\
\hline \multicolumn{9}{|c|}{ P-AngII (pg/ml) } \\
\hline With glucose & $4(2-36)$ & $9(2-38)$ & $7(2-34)$ & \multirow{3}{*}{0.394} & $2(1-7)$ & $3(2-10)$ & $5(3-10)$ & \multirow{3}{*}{0.270} \\
\hline Without glucose & $4(2-40)$ & $9(2-45)$ & $8(2-36)$ & & $3(1-15)$ & $4(2-21)$ & $4(2-23)$ & \\
\hline P (GLM-between) & & 0.773 & & & & 0.940 & & \\
\hline \multicolumn{9}{|c|}{ P-Endot (pg/ml) } \\
\hline With glucose & $1.5(1.2-1.7)$ & $1.5(1.4-2.0)$ & $1.7(1.4-1.9)$ & \multirow{3}{*}{0.001} & $1.0(0.7-1.5)$ & $1.2 *(1.0-1.6)$ & $1.1 *(0.9-1.5)$ & \multirow{3}{*}{0.000} \\
\hline Without glucose & $1.3(1.0-1.6)$ & $1.5^{*}(1.3-1.8)$ & $1.6^{*}(1.3-1.8)$ & & $1.1(0.8-1.5)$ & $1.2 *(0.8-1.8)$ & $1.3(0.9-1.6)$ & \\
\hline P (GLM-between) & & 0.522 & & & & 0.541 & & \\
\hline
\end{tabular}

Data presented as medians with quartiles in brackets. Hormones were measured in the last dialysis session in Week 2 and Week 4 . Blood samples were drawn at dialysis start, after two hours and at the end of the session. A General Linear Model (GLM) for Repeated Measures was used for comparison within and between groups. Wilcoxon's signed rank test was used in each group to analyze significant deviations from baseline $(*=\mathrm{p}<0.05)$. 
Comparison between Type 1 and Type 2 diabetics showed no significant differences in the level of PRC, p-AngII and p-Endot either during dialysis with or without glucose added to dialysis fluid, using a General Linear Model with parameter estimates (PRC: $p=0.803$, p-AngII: $p=0.359$, p-Endot: $\mathrm{p}=0.092)$.

\subsection{Plasma Concentrations of Insulin, Glucagon, and Human Growth Hormone}

Table 5 shows the results for non-diabetics and diabetics.

Neither non-diabetics nor diabetics p-Ins was signifycantly changed during dialysis either with or without glucose. P-Glu was not significantly changed during dialysis with or without glucose in non-diabetics. In diabetics, a marginal increase was measured during dialysis with glucose, but not without glucose. P-hGH decreased significantly during dialysis both with and without glucose in both non-diabetics and diabetics from a level around 3 - $4 \mu \mathrm{U} / \mathrm{ml}$ to $0.3-0.4 \mu \mathrm{U} / \mathrm{ml}$ after both 2 and 4 hours of dialysis ( $\mathrm{p}<0.000$ for both non-diabetics and diabetics).

Both in Type 1 and Type 2 diabetics, p-Ins and p-Glu were unchanged during dialysis treatment with and without glucose and at the same level, but p-hGH fell significantly in both types (Table 6).

Comparison between Type 1 and Type 2 diabetics showed a significantly higher p-Ins in Type 1 compared with Type 2 diabetics during dialysis with or without glucose added to dialysis fluid, using a General Linear Model with parameter estimates (Between Type 1 and 2: $p=0.011$; parameter estimates: at dialysis start: $\mathrm{p}=0.092$, after two hours dialysis: $p=0.040$, and at the end of dialysis $p=0.016$ ).

P-Glu was lower in Type 1 than Type 2 diabetics dur- ing dialysis with or without glucose added to dialysis fluid, using a the same analysis (Between Type 1 and 2: $\mathrm{p}=0.018$; parameter estimates: at dialysis start: $p=0.009$ after two hours dialysis: $\mathrm{p}=0.035$, and at the end of dialysis $\mathrm{p}=$ 0.030).

P-hGH was the same in Type 1 than Type 2 diabetics during dialysis with or without glucose added to dialysis fluid, using a the same analysis (Between Type 1 and 2: $p=0.177$; parameter estimates: at dialysis start: $p=0.159$ after two hours dialysis: $\mathrm{p}=0.851$, and at the end of dialysis $\mathrm{p}=0.130)$.

\subsection{Energy Transfer during Dialysis}

Table 1 shows that the energy transfer was similar during dialysis in both non-diabetics (with glucose: -123 ((-174) $(-43) \mathrm{KJ}$, and without glucose: $-125((-163)-(-73) \mathrm{KJ}), \mathrm{p}$ $=0.494)$, and diabetics (with glucose: $-91(-(117)-(-91)$ ) KJ, and without: $-77((-102)-(-8) \mathrm{KJ}), \mathrm{p}=0.609)$. However, the energy transfer was significantly higher in diabetics than non-diabetics during dialysis without glucose, whereas no significant difference was measured between the two groups with glucose added to the dialysis fluid.

\subsection{Effect of Temperature Control during Dialysis}

Using dialysis treatment with temperature control we aimed to keep the patient's body temperature at the same level during the dialysis session. Systolic blood pressure was significantly higher and the energy loss more pronounced using dialysis with temperature control compared with dialysis without temperature control in a group of nondiabetics (Group 3) as indicated in Table 7. Both body

Table 5. Plasma concentrations of insulin (p-Ins), glucagon (p-Glu), and human growth hormone (p-GH) during hemodialysis treatment using dialysis fluid with glucose (active treatment) and without glucose (placebo) in non-diabetic nephropathy and diabetic nephropathy in a randomized, placebo controlled, crossover study during four consecutive weeks (Week 1: run-in; Week 2: active treatment or placebo; Week 3: wash-out; Week 4: active treatment or placebo).

\begin{tabular}{|c|c|c|c|c|c|c|c|c|}
\hline & \multicolumn{4}{|c|}{ Non-diabetic nephropathy (N = 19) } & \multicolumn{4}{|c|}{ Diabetic nephropathy (N = 15) } \\
\hline & Start & After 2 hours & End & $\begin{array}{c}\mathrm{P} \\
\text { (GLM-with-in) }\end{array}$ & Start & After 2 hours & End & $\begin{array}{c}\mathrm{P} \\
\text { (GLM-with-in) }\end{array}$ \\
\hline \multicolumn{9}{|c|}{ P-Ins $(\mu \mathrm{U} / \mathrm{ml})$} \\
\hline With glucose & $21(9-34)$ & $20(13-43)$ & $23(16-35)$ & \multirow{2}{*}{0.430} & $27(18-35)$ & $22(16-55)$ & $23(14-64)$ & \multirow{3}{*}{0.853} \\
\hline Without glucose & $16(10-34)$ & $11(9-32)$ & $15(9-23)$ & & $31(13-45)$ & $24(16-40)$ & $22(14-35)$ & \\
\hline P (GLM-between) & & 0.083 & & & & 0.972 & & \\
\hline \multicolumn{9}{|c|}{ P-Glu (pg/ml) } \\
\hline With glucose & $203(159-256)$ & $185(141-363)$ & $198(122-364)$ & \multirow{2}{*}{0.446} & $154(145-334)$ & $164(106-275)$ & $157 *(101-256)$ & \multirow{3}{*}{0.048} \\
\hline Without glucose & $211(170-285)$ & $212(138-274)$ & $254(142-308)$ & & $153(124-249)$ & $126(104-265)$ & 146 (99 - 297) & \\
\hline P (GLM-between) & & 0.948 & & & & 0.854 & & \\
\hline \multicolumn{9}{|c|}{ P-hGH $(\mu \mathrm{IU} / \mathrm{ml})$} \\
\hline With glucose & $4.1(2.6-6.4)$ & $0.3^{*}(0.2-0.7)$ & $0.3 *(0.1-1.2)$ & \multirow{2}{*}{0.000} & $3.8(1.7-5.5)$ & $0.3 *(0.2-0.8)$ & $0.2 *(0.1-0.4)$ & \multirow{2}{*}{0.000} \\
\hline Without glucose & $2.6(1.1-4.9)$ & $0.3 *(0.2-0.6)$ & $0.3^{*}(0.1-0.5)$ & & $1.8(1.8-5.5)$ & $0.4 *(0.2-1.1)$ & $0.2 *(0.1-0.6)$ & \\
\hline P (GLM-between) & \multicolumn{4}{|c|}{0.104} & \multicolumn{3}{|c|}{0.742} & \\
\hline
\end{tabular}

Data presented as medians with quartiles in brackets. Hormones were measured in the last dialysis session in Week 2 and Week 4 . Blood samples were drawn at dialysis start, after two hours and at the end of the session. A General Linear Model (GLM) for Repeated Measures was used for comparison within and between groups. Wilcoxon's signed rank test was used in each group to analyze significant deviations from baseline $(*=\mathrm{p}<0.05)$. 
Table 6. Plasma concentrations of insulin (p-Ins), glucagon (p-Glu), and human growth hormone (p-GH) during hemodialysis treatment using dialysis fluid with glucose (active treatment) and without glucose (placebo) in non-diabetic nephropathy and diabetic nephropathy in a randomized, placebo controlled, crossover study during four consecutive weeks (Week 1: run-in; Week 2: active treatment or placebo; Week 3: wash-out; Week 4: active treatment or placebo).

\begin{tabular}{|c|c|c|c|c|c|c|c|c|}
\hline & \multicolumn{4}{|c|}{ Type 1 Diabetic nephropathy ( $\mathrm{N}=8$ ) } & \multicolumn{4}{|c|}{ Type 2 Diabetic nephropathy $(\mathrm{N}=7)$} \\
\hline & Start & After 2 hours & End & $\begin{array}{c}\mathrm{P} \\
\text { (GLM-with-in) }\end{array}$ & Start & After 2 hours & End & $\begin{array}{c}\mathrm{P} \\
\text { (GLM-with-in) }\end{array}$ \\
\hline \multicolumn{9}{|c|}{ P-Ins $(\mu \mathrm{U} / \mathrm{ml})$} \\
\hline With glucose & $28(13-70)$ & $39(18-97)$ & $43(12-98)$ & \multirow{2}{*}{0.234} & $27(18-31)$ & $22(16-24)$ & $22(14-31)$ & \multirow{3}{*}{0.113} \\
\hline Without glucose & $42(8-89)$ & $37(25-82)$ & $29(18-102)$ & & $29(17-31)$ & $16(10-24)$ & $15(13-23)$ & \\
\hline P (GLM-between) & & 0.897 & & & & 0.581 & & \\
\hline \multicolumn{9}{|c|}{ P-Glu (pg/ml) } \\
\hline With glucose & $146(123-185)$ & $114(92-160)$ & $118(92-161)$ & \multirow{2}{*}{0.382} & $208(154-335)$ & $199(164$ - 314) & $195(157-270)$ & \multirow{2}{*}{0.127} \\
\hline Without glucose & $125(110-151)$ & $110(94-171)$ & $118(83-199)$ & & $246(155-351)$ & $248(126-288)$ & $232(146-331)$ & \\
\hline P (GLM-between) & & 0.705 & & & & 0.989 & & \\
\hline \multicolumn{9}{|c|}{ P-hGH $(\mu \mathrm{IU} / \mathrm{ml})$} \\
\hline With glucose & $4.4(1.8-5.4)$ & $0.3^{*}(0.2-1.2)$ & $0.3^{*}(0.1-1.5)$ & \multirow{2}{*}{0.001} & $2.6(0.7-5.5)$ & $0.4(0.1-0.8)$ & $0.1 *(0.1-0.3)$ & \multirow{2}{*}{0.000} \\
\hline Without glucose & $3.8(1.8-9.5)$ & $0.7 *(0.3-2.2)$ & $0.3^{*}(0.1-0.4)$ & & $2.2(0.7-5.5)$ & $0.4(0.2-0.7)$ & $0.2 *(0.1-0.6)$ & \\
\hline P (GLM-between) & \multicolumn{3}{|c|}{0.522} & \multicolumn{5}{|c|}{0.787} \\
\hline
\end{tabular}

Data presented as medians with quartiles in brackets. Hormones were measured in the last dialysis session in Week 2 and Week 4. Blood samples were drawn at dialysis start, after two hours and at the end of the session. A General Linear Model (GLM) for Repeated Measures was used for comparison within and between groups. Wilcoxon's signed rank test was used in each group to analyze significant deviations from baseline $(*=\mathrm{p}<0.05)$.

Table 7. Systolic and diastolic blood pressure (SBP, DBP), pulse rate (PR), body temperature (Body Temp) and energy transfer during hemodialysis treatment using dialysis fluid with glucose in non-diabetic nephropathy $(\mathrm{N}=24)$ with and without temperature control of dialysis fluid in a randomized, placebo controlled, crossover study during two consecutive weeks.

\begin{tabular}{lccc}
\hline & With temperature control of dialysis fluid & Without temperature control of dialysis fluid & $\mathrm{p}$ \\
\hline SBP (mm Hg) & $145(127-156)$ & $138(126-150)$ & 0.036 \\
DBP (mm Hg) & $70(63-74)$ & $67(63-76)$ & 0.868 \\
PR (beats/min) & $71(64-76)$ & $72(67-76)$ & 0.023 \\
Body Temp $\left({ }^{\circ} \mathrm{C}\right)$ & $36.6(36.5-36.8)$ & $36.7(36.5-36.7)$ & 0.087 \\
Energy Transfer $(\mathrm{kJ})$ & $-225((-300)-(-184))$ & $-140((-225)-(-140))$ & 0.003 \\
\hline
\end{tabular}

SBP, DBP, PR and Body Temp are presented as medians with quartiles in brackets. For each dialysis session mean was calculated based on 4 measurements. Mean values of these variables from the three weekly dialysis sessions are indicated. Energy transfer was calculated using a Blood Temperature Monitor which was an accessory to the dialysis machine. Wilcoxon's signed rank test was used for statistical analysis.

temperature and pulse rate were slightly lower during dialysis with temperature control than without, but the differences were very small. Diastolic blood pressure was unchanged by temperature control.

\section{Discussion}

In the present randomized, placebo-controlled, cross-over trial, we measured the effect of adding glucose to the dialysis fluid on blood pressure and vasoactive hormones in non-diabetics and diabetics with chronic renal failure during hemodialysis treatment. The study showed that blood pressure remained unchanged during dialysis with glucose added to the dialysis fluid. Plasma levels of renin and angiotensin II did not change and endothelin increased, but adding glucose to the dialysis fluid did not influence the pattern of vasoactive hormones in plasma during the dialysis sessions. In addition, we measured the effect of temperature control of the dialysis fluid on blood pressure in non-diabetics hemodialysis patients during the dialysis sessions, and we found that systolic blood pressure increased with temperature control of the dialysis fluid.

The present study showed that both systolic and diastolic BP were the same in dialysis patients with or without glucose added to the dialysis fluid. However, in a previous study we measured a decrease in SBP and DBP during dialysis with glucose added to the dialysis fluid [10]. This discrepancy might be attributed to differences in design. In the present study, we used dialysis without glucose added to the dialysis fluid for a period of one week, whereas ten weeks periods were used in our previous study. Most likely, a longer time period is demanded 
for adjustment of the systemic hemodynamics to changes in the content of glucose in the dialysis fluid.

We did not use an automatic blood volume control monitor in the present study, because it would imply that ultrafiltration rate would be adjusted of the hemodialysis machine automatically, using an algorithm based on measurements of red blood cell volume and a feedback loop. Consequently, it must be expected that the incidence of episodes with hypovolemia and fall in blood pressure would be reduced, and a possible effect of glucose added to the dialysis fluid per se would be masked.

The addition of glucose to dialysis fluid had been a controversial issue. Among the advantages were prevention of both glucose loss and a decrease in respiratory quotient, decreased risk of both hypoglycemia and incidence of headache and post-dialysis fatigue, and donation of energy to patients [3-6,7-9]. Among the disadvantages were increased costs, decreased potassium elimination, and augmentation of risk for bacterial growth in liquid bicarbonate concentrates. For the time being, glucose is routinely added to dialysis fluid in our department, but this treatment modality was not a routine praxis, when we performed the previous study [10]. In the present study, patients received dialysis treatment with glucose added to the dialysis fluid, when they entered the study. Thus, their blood pressure regulation was adjusted to glucose added to the dialysis fluid in advance, and it was not changed during a dialysis period of one week duration without glucose added to the dialysis fluid.

In the present study, we measured an increased in pulse rate in diabetics during dialysis treatment without glucose. The reason for this increase is unknown, but it is tempting to speculate that it might be due to an increased sympathetic adrenergic activity induced by the tendency to hypoglycemia, when dialysis is performed without glucose added to the dialysis fluid.

The activity in the renin-angiotensin system was the same in both non-diabetics and diabetics, and it was not significantly changed during dialysis. Although some measurements of PRC and p-AngII were higher than normal range, most values were normal and generally the activeity in the renin-angiotensin system was normal. Several antihypertensive agents influence the activity of the reninangiotensin system, especially ACE-inhibitors and angiotensin II receptor blockers. Many of the patients received such treatment, and most ideal, these drugs should have been discontinued before and during the study. However, we did not find it ethically justified to withdrawn antihypertensive treatment in these patients, but the patients received the same medication and in the same doses during the whole study period.

P-Endot increased during dialysis, and the measured values were higher in dialysis patients than in healthy con- trol subjects. Since endothelin is a very potent vasoconstrictor agent, it is possible that the increased level of this hormone contributed to maintain blood pressure during dialysis, but the increase in p-endot was not influenced by glucose added to the dialysis fluid. Neither was any difference measured between non-diabetics and diabetics nor between type 1 and type 2 diabetics.

In non-diabetics and diabetics, b-glucose was higher during dialysis with glucose in the dialysis fluid than without, and the levels were the same in both groups. However, p-Ins changed differently during dialysis in non-diabetics and diabetics. Thus, in non-diabetics p-Ins remained at a constant level during dialysis with and without glucose, but the level tended to be reduced during treatment without glucose. Most likely, this phenomenon could be attributed to an adequate response of the non-diabetics on glucose added to the dialysis fluid. In the whole group of diabetics, p-Ins was the same during dialysis with and without glucose, but differences existed between Type 1 and Type 2 diabetics. P-Ins was unchanged in Type 1 diabetes and at the same level during dialysis with and without glucose, whereas p-Ins decreased significantly at the end of dialysis in Type 2 diabetes during dialysis without glucose, reflecting some ability to adequate blood glucose regulation in this group. Some of the patients, especially those suffering from type 1 diabetes, had a higher p-Ins than healthy controls due to the fact that they received insulin treatment.

P-Glu was similar in non-diabetics and diabetics, although a tendency to a lower level was measured in diabetics. P-Glu was not significantly changed during dialysis with or without glucose in the two groups. Differences existed, however, between the two subgroups of diabetics. P-Glu was at a significantly lower level in Type 1 diabetes and Type 2 both during dialysis with and without glucose. Thus, the difference is unrelated to dialysis treatment and might reflect a difference in metabolism in chronic renal failure between the two types of diabetes or differences in secretion pattern of incretin hormones, i.e. glucose-dependent insulinotropic polypeptide and glucagon-like peptide-1 $[15,16]$.

The present study showed, that p-hGH was in the lower normal range in both non-diabetics and diabetics with chronic renal failure, and p-hGH was markedly reduced during the dialysis sessions independently of addition of glucose to the dialysis fluid, and no difference was measured between type 1 and type 2 diabetics. The kidney is the major site of growth hormone degradation, and the metabolic clearance rate of growth hormone was reduced in end stage renal failure [17-19]. Fasting p-hGH was elevated in some studies in chronic renal failure, but the response to glucose was not affected [20]. We cannot explain this discrepancy with the results from the present 
study. The pronounced decrease in p-hGH during the dialysis sessions might be due to elimination during dialysis, since the molecule weight is 22.000 Dalton, or to adhesion to the filter membrane, but the mechanism is unknown.

In a separate group comprising 24 non diabetic dialysis patients, we measured a significant increase in SBP using dialysis with temperature control of dialysis fluid compared with dialysis without temperature control (145 versus $138 \mathrm{~mm} \mathrm{Hg}$ ). In parallel with the increase in SBP, the energy flux from the patients was significantly higher with temperature control than without. Most likely, the increase in SBP could be attributed to peripheral vasoconstriction secondary to loss of energy in form of heat. However, the measured body temperature did not deviate significantly between the two dialysis modalities. It is reasonable to suppose that the auto regulatory capacity of body temperature antagonized and overruled the attempt of temperature control during the dialysis procedure. The price for temperature control was a slight increase in SBP and a marginal although significant reduction in pulse rate. Thus, the use of temperature control of dialysis fluid does not seem to be justified on a routinely basis, but can be one of several treatment options to prevent blood pressure fall during dialysis sessions.

To sum up, in non-diabetics and diabetics, blood pressure was unchanged during dialysis with glucose added to the dialysis fluid in a short-term study. Vasoactive hormones in plasma changed in the same way independently of glucose in the dialysis fluid. Systolic blood pressure increased using dialysis with temperature control of dialysis fluid, presumably due to vasoconstriction to prevent or antagonize a fall in body temperature.

\section{Acknowledgements}

The study was supported by Ringkjoebing Amt and Region Midt, Denmark, and by a grant from European Nephrology Dialysis Institute, Oberursel, Germany.

We thank the staff of nurses in the Dialysis Unit and the Laboratory Technicians in Department of Medical Research for skillful assistance to perform the study.

\section{References}

[1] M. A. Jackson, M. R. Holland, J. Nicholas, R. Lodwick, D. Forster and I. A. Macdonald, "Hemodialysis-Induced Hypoglycemia in Diabetic Patients,” Clinical Nephrology, Vol. 54, No.1, 2000, pp. 30-34.

[2] M. A. Jackson, M. R. Holland, J. Nicholas, et al., "Occult Hypoglycemia Caused by Hemodialysis,” Clinical Nephrology, Vol. 51, No. 4, 1999, pp. 242-247.

[3] R. A. Ward, M. J. Shirlow, J. M. Hayes, G. V. Chapman and P. C. Farrell, "Protein Catabolism during Hemodialy- sis," American Journal of Clinical Nutrition, Vol. 32, No. 12, 1979, pp. 2443-2449.

[4] R. A. Ward, R. L. Wathen, T. E. Williams and G. B. Harding, "Hemodialysate Composition and Intradialytic Metabolic, Acid-Base and Potassium Changes,” Kidney International, Vol. 32, 1987, pp. 129-135. doi:10.1038/ki.1987.182

[5] A. Gutierrez, J. Bergstrom and A. Alvestrand, "Hemodialysis-Associated Protein Catabolism with and without Glucose in the Dialysis Fluid,” Kidney International, Vol. 46, 1994, pp. 814-822. doi:10.1038/ki.1994.337

[6] C. L. Skutches and M. H. Sigler, "Plasma Glucose Turnover and Oxidation during Hemodialysis: Nutritional Effect of Dialysis Fluid," American Journal of Clinical Nutrition, Vol. 65, No. 1, 1997, pp. 128-135.

[7] S. F. Raju, A. R. White, T. T., Barnes P. P. Smith and K. A. Kirchner, "Improvement in Disequilibrium Symptoms during Dialysis with Low Glucose Dialyzate,” Clinical Nephrology, Vol. 18, No. 3, 1982, pp. 126-129.

[8] M. Leski, T. Niethammer and T. Wyss, "Glucose-Enriched Dialysate and Tolerance to Maintenance Hemodialysis,” Nephron, Vol. 24, No. 6, 1979, pp. 271-273. doi:10.1159/000181734

[9] G. Ramirez, B. L. Bercaw, D. E. Butcher, H. L. Mathis, C. Brueggemeyer and J. L. Newton, "The Role of Glucose in Hemodialysis: The Effects of Glucose-Free Dialysate," American Journal of Kidney Diseases, Vol. 7, No. 5, 1986, pp. 413-420.

[10] M. Sangill and E. B. Pedersen, "The Effect of Glucose Added to the Dialysis Fluid on Blood Pressure, Blood Glucose and Quality of Life in Hemodialysis Patients: A Placebo Controlled Cross-Over Study," American Journal of Kidney Diseases, Vol. 47, No. 4, 2006, pp. 636-643. doi:10.1053/j.ajkd.2006.01.007

[11] A. D. Baron, "Hemodynamic Actions of Insulin," American Journal of Physiology, Vol. 267, No. 2, 1994, pp. E187-202.

[12] J. E. Hall, T. G. Coleman, H. L. Mizelle and M. J. Smith Jr., "Chronic Hyperinsulinemia and Blood Pressure Regulation,” American Journal of Physiology, Vol. 258, No. 3, 1990, pp. F722-731,

[13] R. A. DeFronzo, J. D. Tobin, J. W. Rowe and R. Andres, "Glucose Intolerance in Uremia. Quantification of Pancreatic Beta Cell Sensitivity to Glucose and Tissue Sensitivity to Insulin,” Journal of Clinical Investigation, Vol. 62, 1978, pp. 425-435. doi:10.1172/JCI109144

[14] E. B. Pedersen, H. Eiskjaer, B. Madsen, H. Danielsen, M. Egeblad and C. B. Nielsen, ”Effect of Captopril on Renal Extraction of Renin, Angiotensin II, Atrial Natriuretic Peptide and Vasopressin, and Renal Vein Renin Ratio In Patients with Arterial Hypertension and Unilateral Renal Artery Disease," Nephrology Dialysis Transplantation, Vol. 8, No. 10, 1993, pp. 1064-1070.

[15] S. Efendic and N. Portwood, "Overview of Incretin Hormones,” Hormone and Metabolic Research, Vol. 36, No. 11-12, 2004, pp. 742-746. doi:10.1055/s-2004-826157

[16] D. J. Drucker, “The Role of Gut Hormones in Glucose 
Homeostasis,” Journal of Clinical Investigation, Vol. 117, No. 1, 2007, pp. 24-32. doi:10.1172/JCI30076

[17] V. Johnson and T. Maack, "Renal Extraction, Filtration, Absorption, and Catabolism of Growth Hormone," American Journal of Physiology, Vol. 233, No. 3, 1977, pp. F185-196.

[18] D. Haffner, F. Schaefer, J. Girard, E. Ritz and O. Mehls, "Metabolic Clearance of Recombinant Human Growth Hormone in Health and Chronic Renal Failure," Journal of Clinical Investigation, Vol. 93, No. 3, 1994, pp. 11631171. doi:10.1172/JCI117069
[19] J. D. Veldhuis, A. Iranmanesh, M. J. Wilkowski and E. Samojlik, "Neuroendocrine Alterations in the Somatotropic and Lactotropic Axes in Uremic Men,” European Journal of Endocrinology, Vol. 131, No. 5, 1994, pp. 489498. doi:10.1530/eje.0.1310489

[20] V. Allegra, F. Amendolagine, G. Mengozzi, M. Tenti and A. Vasile, "Growth Hormone Secretion Abnormalities in Uremic Patients: Which Is the Role of Impaired Glucose Hypothalamic Sensitivity?” Nephron, Vol. 48, No. 1, 1988, pp. 76-77. doi:10.1159/000184874 